
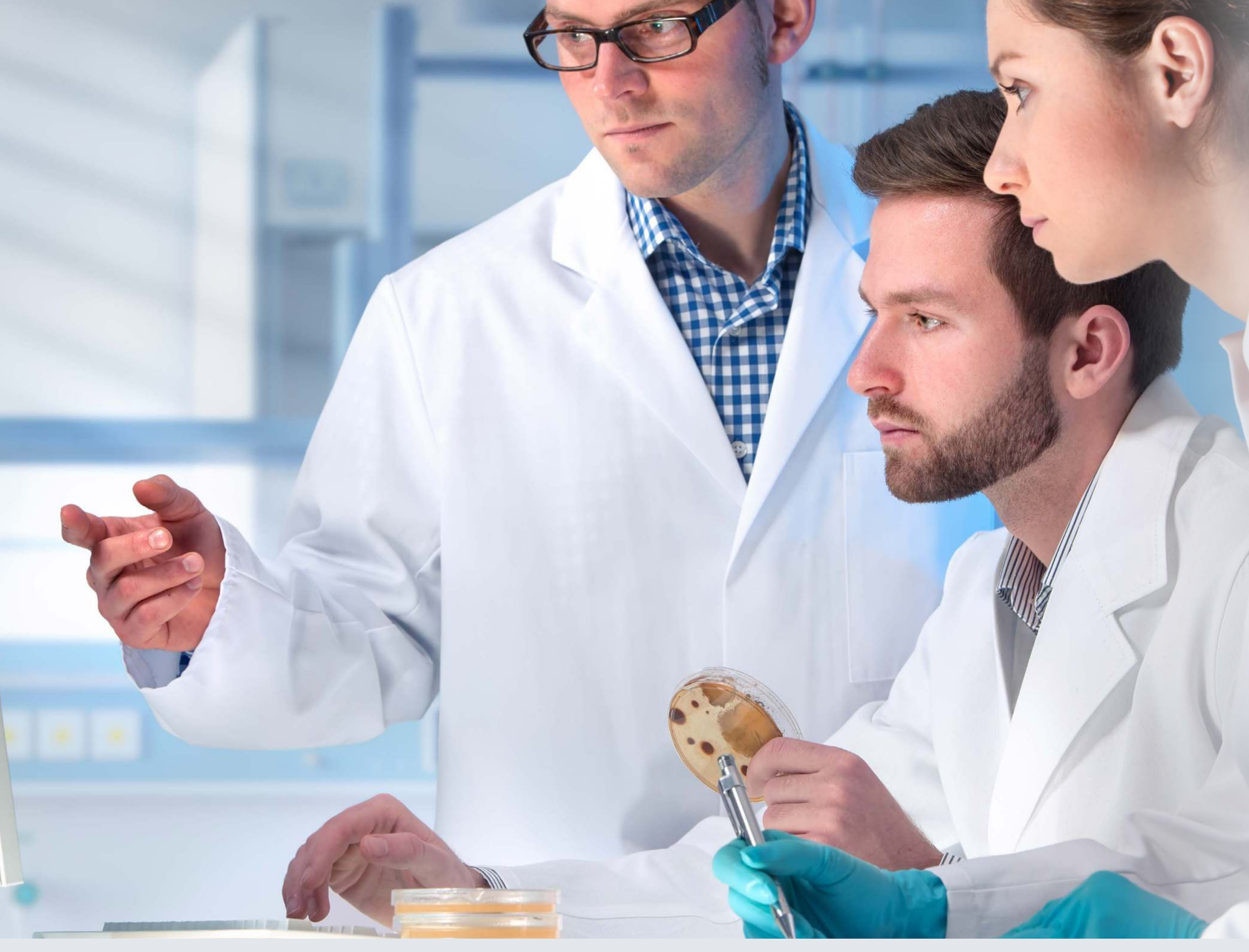

(iD) Peter J. Carr ${ }^{1,2}$ James CR. Rippey ${ }^{1,2,3}$ (iD Marie L Cooke ${ }^{2}$

(iD) Niall S. Higgins ${ }^{247}$

(iD) Michelle Trevenen ${ }^{5}$

Aileen Foale ${ }^{6}$

(D) Claire Rickard ${ }^{24}$

1 School of Medicine, Faculty of Health and Medical Sciences, The University of Western Australia, Nedlands, Australia. 2 Alliance for Vascular Access Teaching \& Research (AVATAR) Group, Menzies Health Institute Queensland, Griffith University, Brisbane, Australia. 3 Sir Charles Gairdner Hospital, QEII Medical Centre, Nedlands, 6 Fiona Stanley Hospital, Murdoch, Australia. 7 Queensland University of Technology, Brisbane, Australia

\section{Introduction}

- The peripheral intravenous catheter/cannula (PIVC) is the most used vascular access device in healthcare today.

- Premature failure PIVC after insertion reveals undesirable rates of failure with $30-50 \%$ of devices failing before the completion of therapy.

- Most strategies are targeted to reduce infection; however despite it being the most harmful to patients it is also the least likely to occur.

- Focusing on the Emergency Department (ED) clinical environment where many patients are exposed to their first hospital PIVC insertion could achieve implementation of improvement strategies at the clinical source.

\section{Methods}

- Design: Prospective multi-centre observational cohort of admitted ED patients.

- PIVC insertions were observed by trained data collectors and were followed up to identify if post insertion failure (PIF) occurred.

The primary outcome was to establish the reasons for removal of the PIVC to identify rates of PIF and dwell times.

Factors considered were: age, gender, skin shade, Australasian Triage Scale (ATS), ultrasound, site of insertion, the inserting clinician's role and the performance of an aseptic non-touch technique keeping key parts protected at insertion (defined as clinical breach or did not breach).
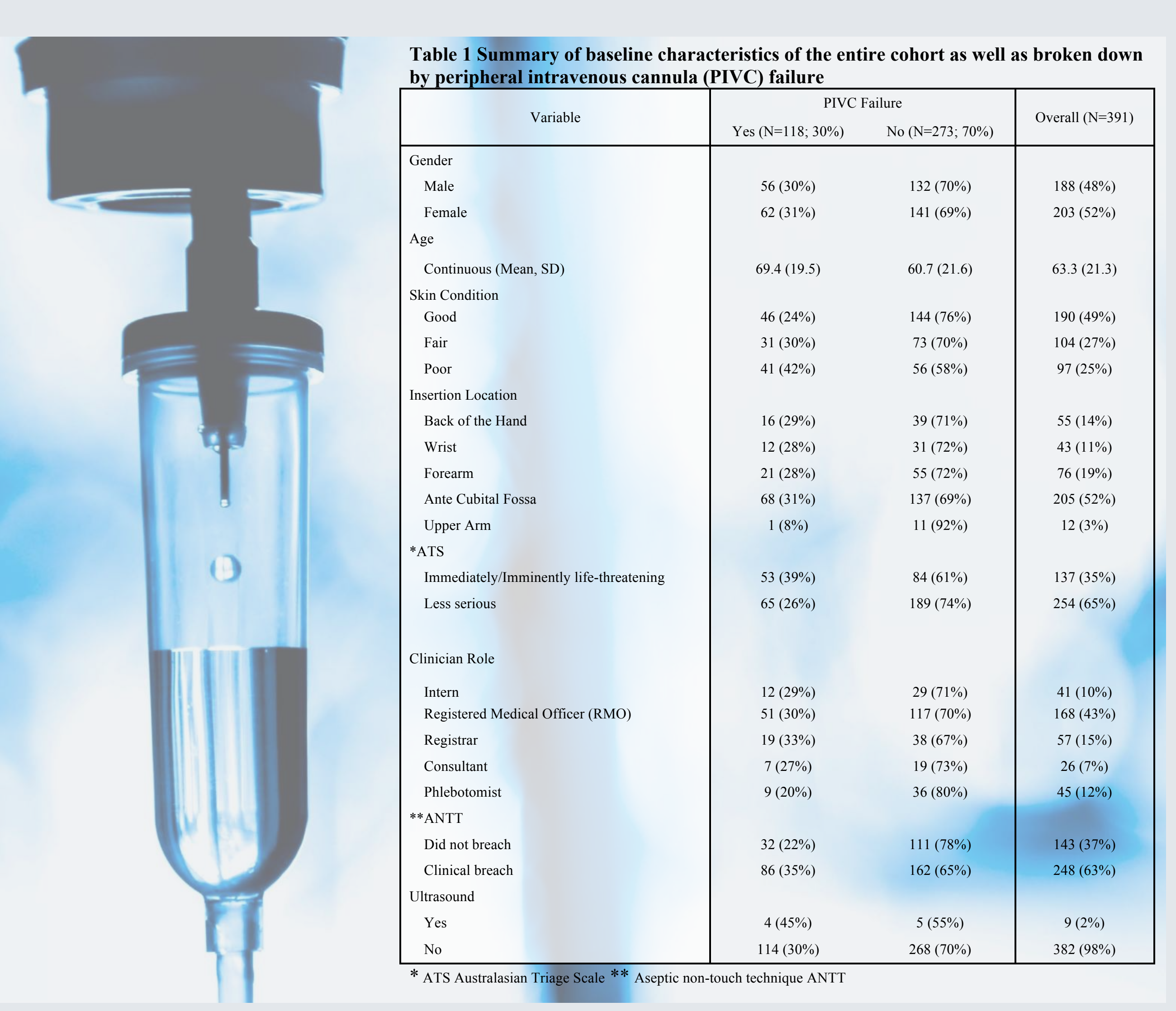

\section{New Knowledge}

- This is the first study to our knowledge to identify PIF is greatest when the PIVC is inserted by medical students.

- PIVC inserted with an aseptic non-touch technique are associated with reduced PIF.



Results

- The rate of PIVC failure was $30 \%$

- The median hospital length of stay was 2 (IQR 1-5) days and the median PIVC dwell-time of 28.5 (IQR 17.4-50.8) hours.

- PIVC failure had a median PIVC dwell time of 24.1 (IQR 11.750.8) hours

- Patients without PIVC failure had a median PIVC dwell time of 29.9 (IQR 20.7-49.8) hours

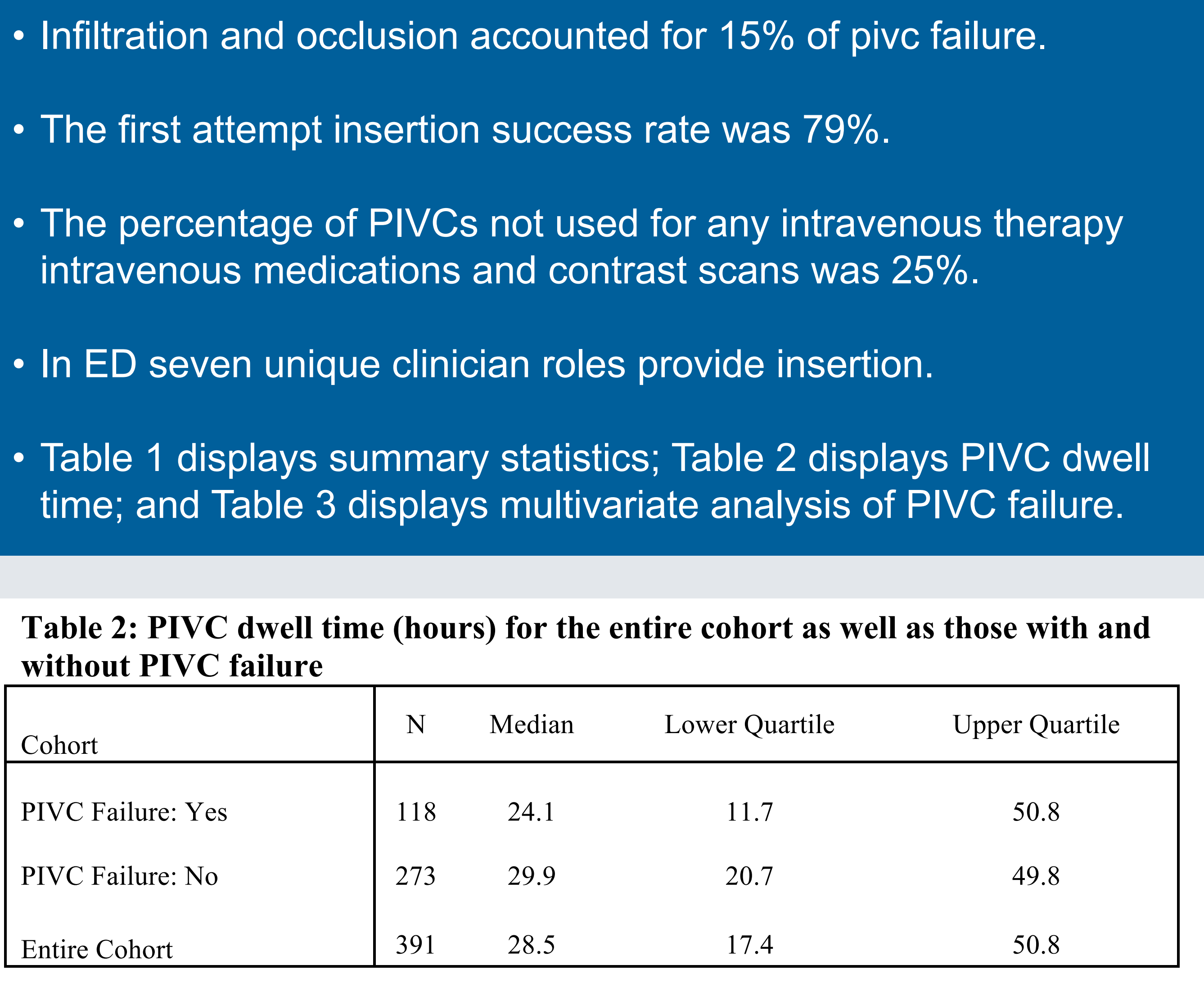

Future Strategies

- Specific PIVC educational interventions for all staff inserting PIVCs and perhaps specifically for medical students to assess the validity of this result.

Targeted educational and workforce strategies should be considered to reduce ATS 1 and 2 patients and USGPIVC experiencing PIF.

\section{FROM INSERTION TO} REMOVAL OF EMERGENCY DEPARTMENT INSERTED PERIPHERAL INTRAVENOUS CATHETERS:

A MULTI-CENTRE SURVIVAL ANALYSIS OF AN ADMITTED COHORT.

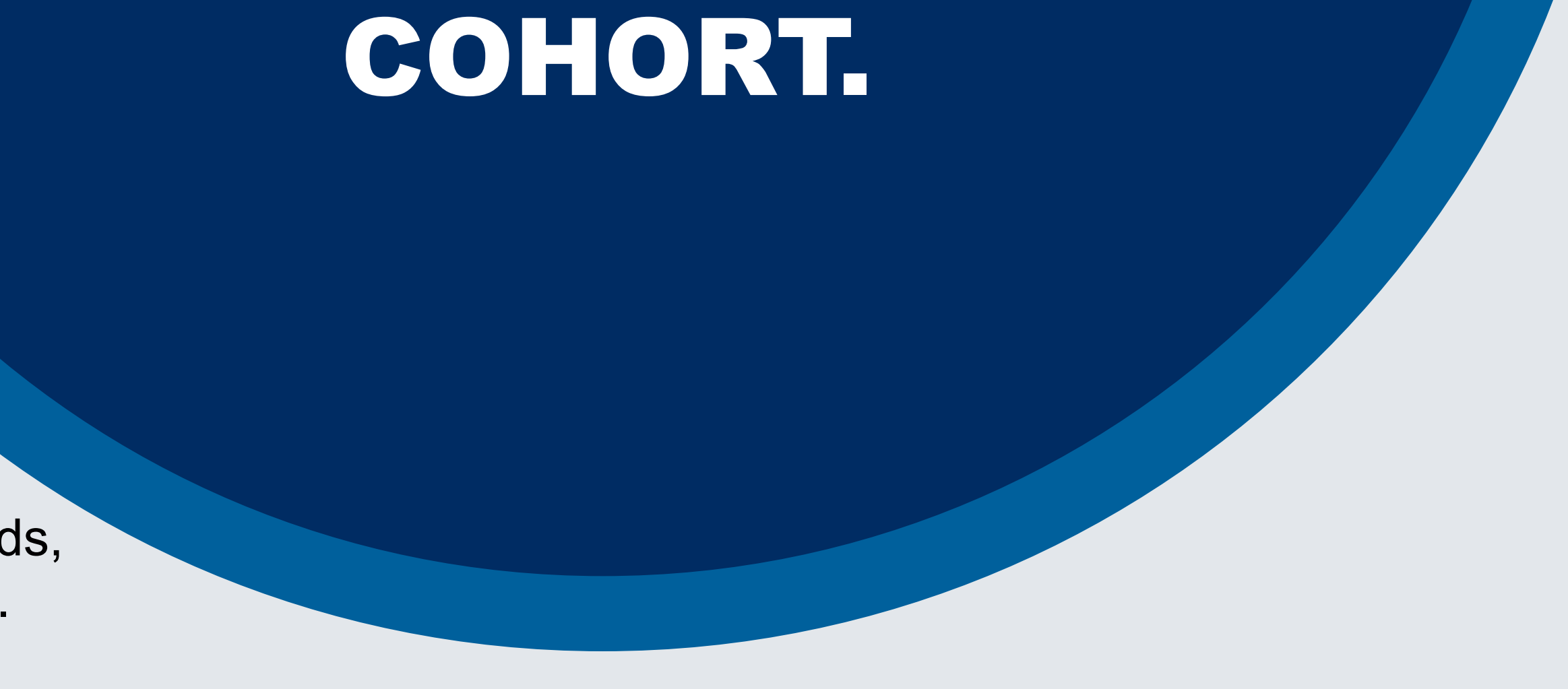

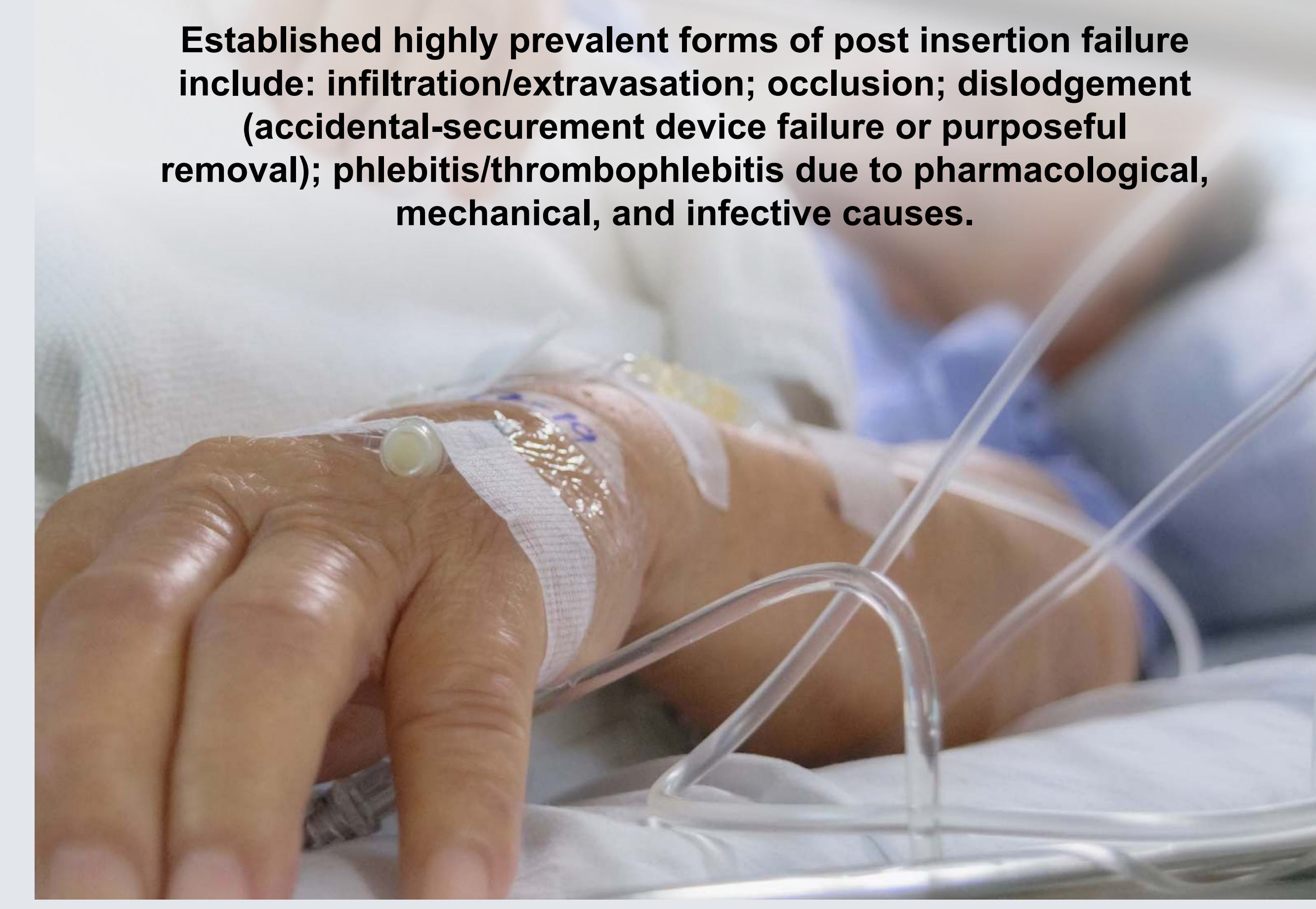

Statistical Analysis

- Forward stepwise model selection was performed where the variable entry and exit significance level was set at $5 \%$.

- Proportional hazards assumption was tested by including the time-dependent covariates in the model and checking for significance.

- Hazard ratios (HR), 95\% confidence intervals (Cl), and P-values are provided.

- Data were analysed using the $R$ environment for statistical computing (version 3.4.4)

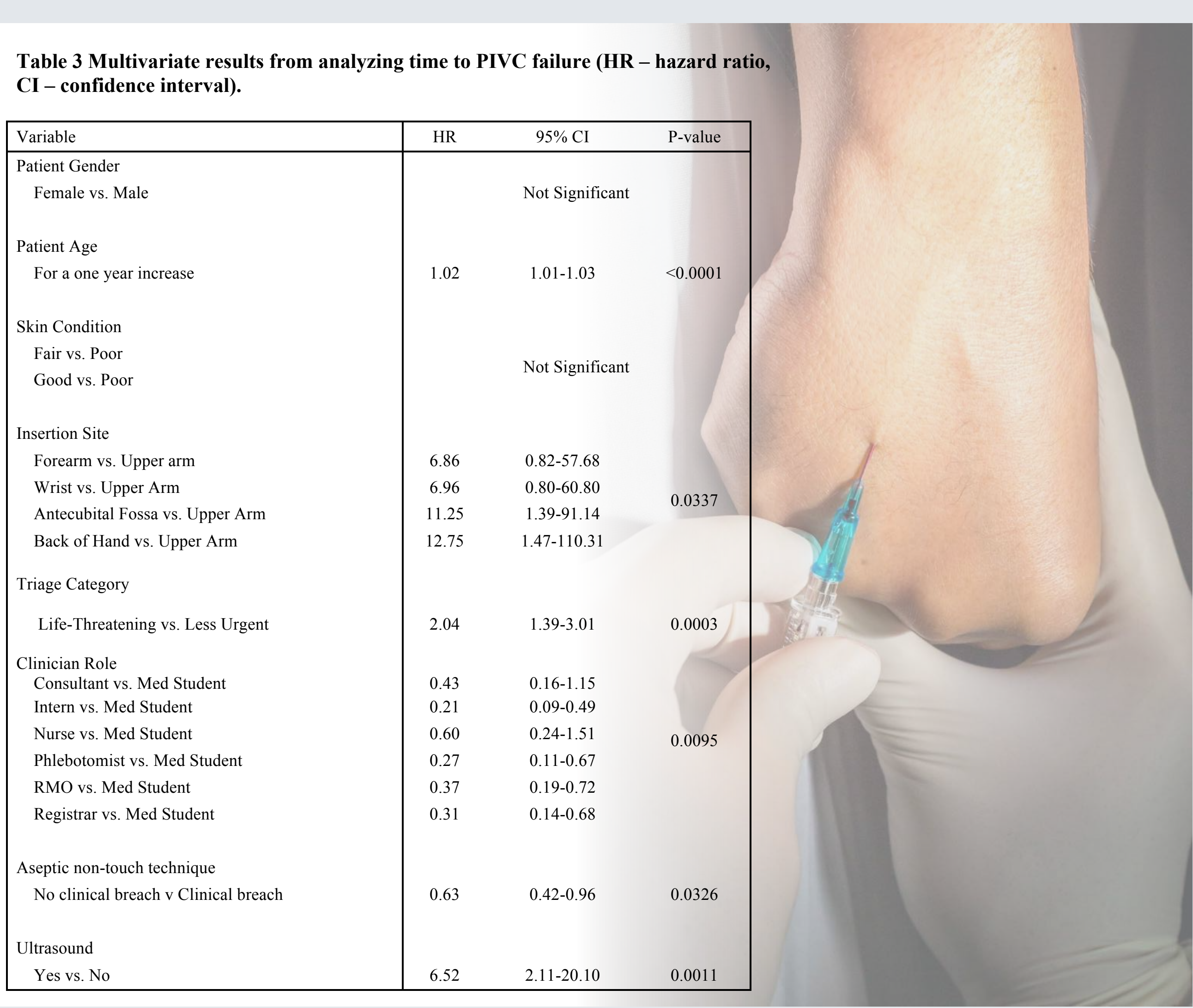

\section{Conclusion}

- Our results of $30 \%$ succumbing to PIF echoes recent reports of similar failure rates all from largely modifiable reasons.

-Where it is clinically achievable the ante cubital fossa region and back of the hand should be avoided in favour of the forearm veins for PIVC placement.

\title{
Clinical Trial Registration
}

Australian and New Zealand Trials Registry (ANZCTRN12615000588594)

AHATAR GROUP \section{แ) Guriffith}

MENZIES

HEALTH INSTITUTE QUEENSLAND 